\title{
Correction to: Considering Methodological Accommodation to the Diversity of ASD: A Realist Synthesis Review of Data Collection Methods for Examining First-Person Experiences
}

\author{
David B. Nicholas $^{1}$ • Jesse D. Orjasaeter ${ }^{1}$ - Lonnie Zwaigenbaum ${ }^{2}$ \\ Published online: 11 March 2019 \\ (C) Springer Science+Business Media, LLC, part of Springer Nature 2019
}

\section{Correction to: Rev J Autism Dev Disord https://doi.org/10.1007/s40489-019-00164-z}

On page 5 in the published version, the following text is incorrectly formatted as a block quote, currently appearing as:

Talking Mats is a visual method that invites participants to (1) reflect and describe the issues which affect their quality of life (not just those perceived by carers and/or professionals) and which matter most, (2) ensure that their specific concerns are acknowledged and dealt with and not just assumed to be caused by their long-term condition, (3) recognize and develop their own strengths and abilities, (4) share their insights relating to their health and social care in an easily accessible and recordable manner across all settings, (5) own and implement self-management solutions, (6) evaluate their progress and the long-term impact of using (this) approach for their self-management, and (7) increase communication with health and social care professionals (Murphy 2016).

The online version of the original article can be found at https://doi.org/ 10.1007/s40489-019-00164-Z

David B. Nicholas

nicholas@ucalgary.ca

1 Faculty of Social Work, Central and Northern Alberta Region, University of Calgary, Edmonton, Canada

2 Department of Pediatrics, Division of Developmental Pediatrics, University of Alberta, Glenrose Rehabilitation Hospital, Edmonton, Canada
Additionally, the subheading for this section has been deleted. The correct text should appear as:

Talking Mats Talking Mats is a visual method that invites participants to:

(1) reflect and describe the issues which affect their quality of life (not just those perceived by carers and/or professionals) and which matter most, (2) ensure that their specific concerns are acknowledged and dealt with and not just assumed to be caused by their long-term condition, (3) recognize and develop their own strengths and abilities, (4) share their insights relating to their health and social care in an easily accessible and recordable manner across all settings, (5) own and implement selfmanagement solutions, (6) evaluate their progress and the long-term impact of using (this) approach for their self-management, and (7) increase communication with health and social care professionals (Murphy 2016).

Please note how only the indented section is a direct quote, and the subheading has been restored. No page number is listed as the citation is for a single webpage.

Acknowledgements We want to acknowledge with gratitude the input of Dr. Mayada Elsabbagh, Ms. Rackeb Tesfaye, Dr. Pat Mirenda, Dr. Stelios Georgiades, and Dr. Peter Szatmari in support of this work. Funding from Kids Brain Health Network and the Canadian Institutes for Health Research is acknowledged.

Publisher's Note Springer Nature remains neutral with regard to jurisdictional claims in published maps and institutional affiliations. 